\title{
Application of an EMCCD camera for calibration of hard X-ray telescopes
}

Julia K. Vogel, Michael J. Pivovaroff, Vivek V. Nagarkar, Haris Kudrolli, Kristin Kruse Madsen, et al.

Julia K. Vogel, Michael J. Pivovaroff, Vivek V. Nagarkar, Haris Kudrolli, Kristin Kruse Madsen, Jason E. Koglin, Charles J. Hailey, William W. Craig, Finn E. Christensen, Nicolai F. Brejnholt, "Application of an EMCCD camera for calibration of hard X-ray telescopes," Proc. SPIE 8443, Space Telescopes and Instrumentation 2012: Ultraviolet to Gamma Ray, 84432L (17 September 2012); doi: 10.1117/12.926386

Event: SPIE Astronomical Telescopes + Instrumentation, 2012, Amsterdam, Netherlands 


\title{
Application of an EMCCD Camera for Calibration of Hard X-Ray Telescopes
}

\author{
Julia K. Vogel ${ }^{a}$, Michael J. Pivovaroff ${ }^{a}$, Vivek V. Nagarkar ${ }^{b}$, Haris Kudrolli ${ }^{b}$, \\ Kristin Kruse Madsen ${ }^{c}$, Jason E. Koglin ${ }^{d}$, Charles J. Hailey ${ }^{d}$, William W. Craig ${ }^{a}$, \\ Finn E. Christensen ${ }^{e}$, and Nicolai F. Brejnholt ${ }^{e}$ \\ ${ }^{a}$ Physical and Life Sciences Department, Lawrence Livermore National Laboratory, CA, USA; \\ ${ }^{b}$ Radiation Monitoring Devices (RMD) Inc., Watertown, MA, USA; \\ ${ }^{c}$ Caltech Division Physics, Mathematics and Astronomy, Pasadena, CA, USA; \\ ${ }^{d}$ Columbia University Astrophysics Laboratory, New York, NY, USA; \\ ${ }^{e}$ National Space Institute (DTU Space), Technical University of Denmark, Copenhagen, \\ Denmark
}

\begin{abstract}
Recent technological innovations make it feasible to construct efficient hard x-ray telescopes for space-based astronomical missions. Focusing optics are capable of improving the sensitivity in the energy range above $10 \mathrm{keV}$ by orders of magnitude compared to previously used instruments. The last decade has seen focusing optics developed for balloon experiments and they are implemented in approved space missions such as the Nuclear Spectroscopic Telescope Array $(N u S T A R)$. The full characterization of x-ray optics for astrophysical missions, including measurement of the point spread function (PSF) as well as scattering and reflectivity properties of substrate coatings, requires a large area detector with very high spatial resolution and sensitivity, photon counting and energy discriminating capability. Novel back-thinned Electron Multiplying Charge-Coupled Devices (EMCCDs) are suitable detectors for ground-based calibrations if combined with a scintillating material. This optical coupling of the EMCCD chip to a microcolumnar CsI(Tl) scintillator can be achieved via a fiberoptic taper. Not only does this detector system exhibit low noise and high spatial resolution inherent to CCDs, but the EMCCD is also able to handle high frame rates. Additionally, thick CsI(Tl) yields high detection efficiency for x-rays. In this paper, we discuss the advantages of using an EMCCD to calibrate hard x-ray optics. We will illustrate the promising features of this detector solution using examples of data obtained during the ground calibration of the NuSTAR telescopes performed at Columbia University during 2010/2011. Finally, we give an outlook on latest development and optimizations.
\end{abstract}

Keywords: EMCCD, NuSTAR, hard x-ray mission, x-ray detectors, calibration, scintillator

\section{INTRODUCTION}

During the last 40 years, observational astronomy has expanded from the relatively narrow wavelength band of visible light to the entire electromagnetic spectrum. X-ray astronomy, covering the band of photon energies from $0.1 \mathrm{keV}$ to several $100 \mathrm{keV}$, belongs to one of the most flourishing of these newly opened spectral ranges. Technological innovations over the last decade in the development of hard x-ray focusing optics are crucial to the advancement of the field of x-ray astronomy at higher energies. Focusing optics provide high sensitivity in the hard x-ray range above $10 \mathrm{keV}$ compared to previously employed devices like collimators and coded-mask cameras. Increases by up to a factor of 100 in sensitivity and a factor of 10 in resolution over the hard x-ray range can be achieved using this technology over conventional devices. Such focusing optics were recently developed for

Further author information: (Send correspondence to J.K.V.)

J.K.V.: E-mail: vogel9@llnl.gov, Telephone: 19254244815

This work was performed under the auspices of the U.S. Department of Energy by Lawrence Livermore National Laboratory under Contract DE-AC52-07NA27344. The support of the Laboratory Directed Research and Development Program is gratefully acknowledged. We also thank NASA for funding this research under grant number NNX11CH32P.

Space Telescopes and Instrumentation 2012: Ultraviolet to Gamma Ray, edited by Tadayuki Takahashi, Stephen S. Murray, Jan-Willem A. den Herder,

Proc. of SPIE Vol. 8443, 84432L - (c) 2012 SPIE · CCC code: 0277-786/12/\$18 - doi: 10.1117/12.926386

Proc. of SPIE Vol. $844384432 \mathrm{~L}-1$ 

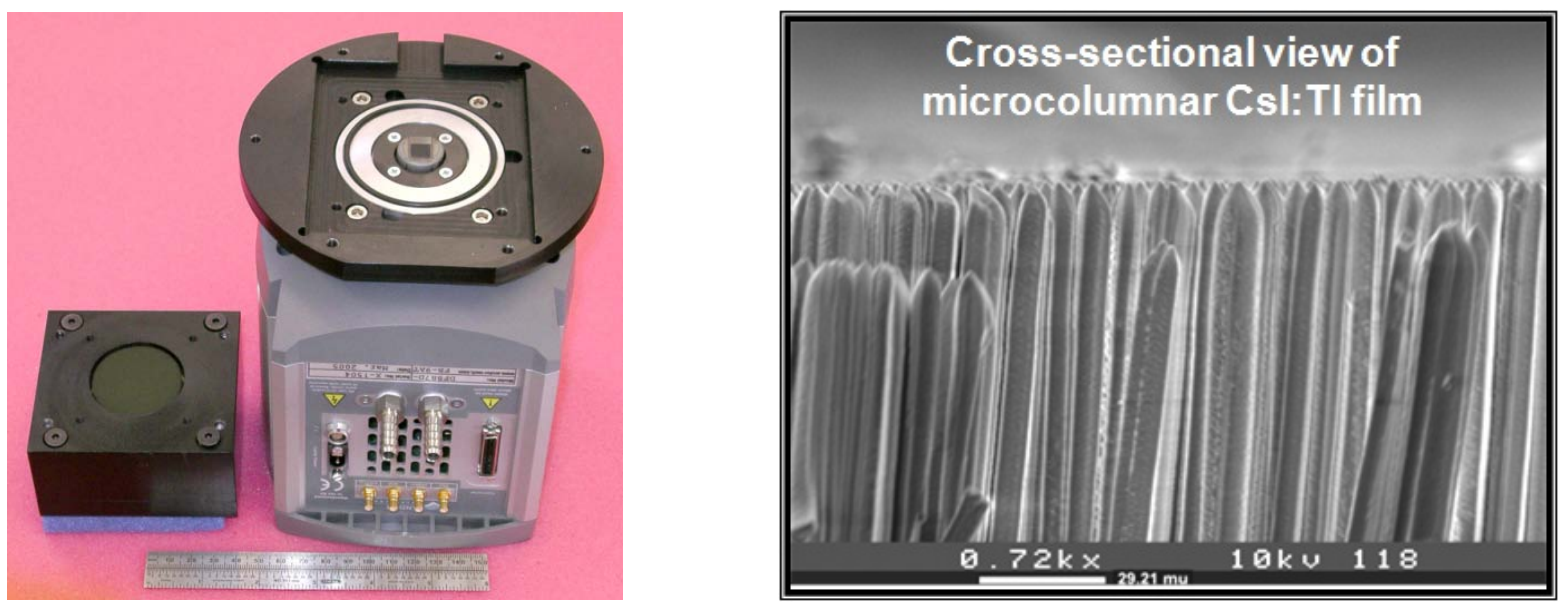

Figure 1. Left: The Andor iXon EMCCD camera developed at RMD. ${ }^{6,7}$ A 1:1 fiberoptic plug is permanently attached to the chip. In the left part of the image an additional, detachable 3:1 fiberoptic taper is shown. Right:Cross-sectional SEM view of microcolumnar $\mathrm{CsI}(\mathrm{Tl})$ film which was deposited on a suitable substrate. ${ }^{8}$ The column diameter is about $10 \mu \mathrm{m}$ on average.

balloon experiments. ${ }^{1-3}$ They are also implemented in current and approved space missions such as the Nuclear Spectroscopic Telescope Array $\left(N u S T A R^{4}\right)$ and ASTRO-H. ${ }^{5}$

Calibration of the focusing components is crucial, on the ground as well as in orbit. A full characterization of the optics includes the measurement of the point spread function and effective area as well as the determination of scattering and reflectivity properties of substrate coatings. This requires a detector with high sensitivity, very high spacial resolution along with good energy discrimination. Such a device should cover a large area and have single-photon counting capability. A prototype detector has already been tested and successfully used in characterizing NuSTAR flight and flight spare telescopes. The analysis of these data demonstrated the advantages of such a 2D detector for hard x-rays $(\gtrsim 25 \mathrm{keV})$. On the other hand it also indicated that there are some limitations for this device in the $5-25 \mathrm{keV}$ range. We will use the $N u S T A R$ calibration data to demonstrate the potential of the detector system for calibrating hard x-ray space missions and present methods to improve the detector performance for calibration of future x-ray telescopes. The features of such an improved detector include high spatial resolution (about $25 \mu \mathrm{m}$ ), large detector area $\left(\sim 5 \times 5 \mathrm{~cm}^{2}\right)$, high sensitivity over the energy range from $5-100 \mathrm{keV}$, and high count rate capability. A flexible design of an easy-to-operate detector (e.g. no need for cryogenic cooling) will make it easier to adapt the detector to various mission requirements.

\section{THE SCINTILLATOR-COUPLED EMCCD DETECTOR}

Conventional CCDs are widely used in x-ray imaging applications since they offer good spatial resolution, high quantum efficiency over the visible range, low noise and a large dynamic range. In high-speed operation, when high frame rates are needed, however, read noise becomes a limiting factor. It is a direct consequence of noise originating from the on-chip output amplifier of the charge-coupled device. Electron Multiplying Charge-Coupled Devices (EMCCDs) were specifically designed to overcome this limitation. In principal, these detectors work like conventional CCDs with the exception of providing internal gain via an avalanche mechanism such that they are suitable for fast x-ray imaging. ${ }^{7}$ Another limiting factor of CCDs is the relatively small active imaging area. A fiberoptic reducer can be used to overcome this limitation.

The detector system presented in this paper consists of an EMCCD, which is back-thinned and optically bonded to a scintillating material that converts the incoming x-rays into visible photons. The bonding of the microcolumnar thallium-doped cesium iodide $(\mathrm{CsI}(\mathrm{Tl}))$ scintillator is accomplished via a coherent fiberoptic taper to increase the active area of the detector. 
Table 1. Overview of features of the Andor EMCCD iXon $887^{6}$

\begin{tabular}{|l|l|}
\hline Active Pixels & $512 \times 512$ \\
\hline Pixel Size & $16 \times 16 \mu \mathrm{m}^{2}$ \\
\hline Image Area (without taper) & $8.2 \times 8.2 \mathrm{~mm}^{2}$ \\
\hline Active Area pixel well depth (typical) & $220,000 \mathrm{e}^{-}$ \\
\hline Gain Register pixel well depth (typical) & $800,000 \mathrm{e}^{-}$ \\
\hline Maximal Readout rate & $10 \mathrm{MHz}$ \\
\hline Frame Rate & 34 to several 100 frames $/ \mathrm{sec}$ \\
\hline Read Noise & $<1$ to $62 \mathrm{e}^{-}$@ $10 \mathrm{MHz}$ \\
\hline Electron Multiplier Gain & $1-1000$ times \\
\hline Peak Quantum Efficiency @-20 ${ }^{\circ} \mathrm{C}($ @575 nm, typical) & $92.5 \%$ \\
\hline
\end{tabular}

\section{The EMCCD Camera}

One of the core pieces of the detector system used during the NuSTAR calibration campaign is an Andor iXon DV887 EMCCD camera. The chip consists of $512 \times 512$ pixels, with a size of $16 \mu \mathrm{m} \times 16 \mu \mathrm{m}$ each, yielding a total active detector area of $67.1 \mathrm{~mm}^{2}$. The EMCCD chip is directly bonded to a 1:1 fiberoptic plug, which is permanently installed to connect the chip and the scintillator material. Since our observation required a larger sensitive area, an additional, detachable 3:1 taper was installed. This increases the detector area by a factor of 9 , but at the same time decreases the sensitivity of the camera due to the optical light loss in the fiber optic taper. Both the EMCCD camera and the 3:1 fiberoptic taper are shown in the left part of Fig. 1, while Tab. 1 summarizes the main features of the EMCCD for the detector prototype.

\section{The CsI(Tl) Scintillator}

The second main component of the detector system is the scintillator used to detect the incoming x-rays and convert them into visible photons detectable with the EMCCD chip. The scintillating material of choice is microcolumnar CsI(Tl). Its properties and low costs together with the existing technologies to deposit it in columnar structure distinguish it from other candidates. The microcolumnar structure makes use of the principle of total internal reflection to guide the scintillation light to the EMCCD chip with minimum light loss and high spatial resolution $\left(10 \mathrm{lp} / \mathrm{mm}\right.$ or more $\left.{ }^{8}\right)$. $\mathrm{CsI}(\mathrm{Tl})$ has a very high conversion efficiency, its initial decay is fast (680 ns), the produced light is in the visible range (about $540 \mathrm{~nm}$ ) and the scintillator is transparent to its own scintillation light. The microcolumnar $\mathrm{CsI}(\mathrm{Tl})$ for this detector was fabricated at RMD Inc. A cross-sectional image obtained using a Scanning Electron Microscope (SEM) is shown in the right part of Fig. 1.

In summary, the advantages of EMCCD detector system as described are multifold. It exhibits both the low noise and high spatial resolution typical for CCDs, but in addition it is also able to handle high frame rates due to the fact that its internal gain is controllable. Furthermore, the use of a high-density and high light-yield scintillator which exhibits a sufficiently fast decay time and can be produced as a film by vapor deposition onto graphite or other suitable substrates is advantageous. High detection efficiencies for x-rays can be achieved by using thick $\mathrm{CsI}(\mathrm{Tl})$ films. Additionally, the active area can be increased by the use of a fiberoptic taper. These advantages, in combination with the robust turn-key technology of the detector, make this device a good candidate for characterizing optics for future hard x-ray space missions.

\section{THE NUSTAR TELESCOPES}

In this section, we briefly introduce the main features of the optics system used for the NuSTAR mission, since we will use examples of the calibration measurements for these optics to demonstrate the performance of the detector system. A more detailed description of the NuSTAR apparatus, its calibration and the science mission can be found in Refs. ${ }^{4}, 13$ 


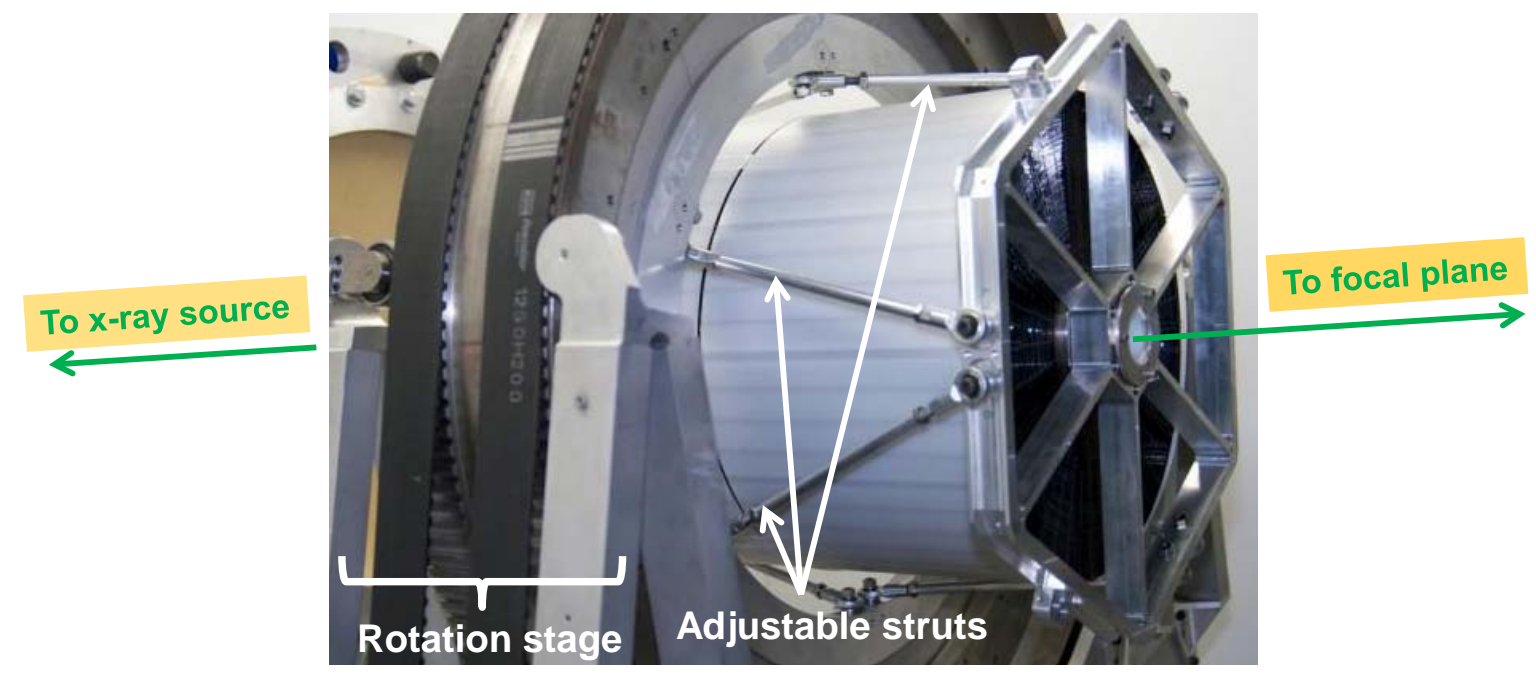

Figure 2. The NuSTAR optic mounted on a rotation stage at the calibration facility. Adjustable struts hold the optic in place and can be regulated for alignment.

NuSTAR is one of NASA's Small Explorer (SMEX) missions and was launched on June 13, 2012. The satellite features two coated multilayer (ML) optics with CdZnTe detectors in the focal plane and is sensitive in the energy band of $5-80 \mathrm{keV}$. The application of ML x-ray mirror optics ${ }^{9,11}$ will enable the state-of-the art experiment to increase its sensitivity by a factor of 50-100 in the hard x-ray regime as compared to previous missions. The sub-arcminute resolution of NuSTAR paired with its excellent spectral resolution will allow for new studies in astrophysics such as the study of supermassive black holes, compact objects in the Galaxy, and supernovae remnants.

The NuSTAR optics (see Fig. 2) are conical approximations to Wolter-I telescopes . Each optic consists of 2340 pieces of glass substrates with multilayer coating. These pieces form 133 shells with individual substrates covering an angle of $60^{\circ}$ for the inner 68 layers, while in the outer layers (layer $69-133$ ) each piece spans an angle of $30^{\circ}$. To optimize the instrument for energies from $5-80 \mathrm{keV}$ the inner 89 layers are coated with $\mathrm{Pt} / \mathrm{C}$, the outer 44 layers with $\mathrm{W} / \mathrm{Si}$. The focal length of the optics is $10.15 \mathrm{~m}$.

The NuSTAR telescopes were calibrated at the Rainwater Memorial Calibration Facility $(R a M C a F)$. During calibration measurements, the telescopes were illuminated in different ways. If the entire telescope aperture was flooded with x-rays, this is commonly referred to as full-flood illumination, while the term subgroup illumination is used to express that only limited regions, i.e. subgroups of the optic, were illuminated by x-rays due to the use of lead plates with narrow, annular openings installed between the x-ray source and the telescope. A detailed description of RaMCaF together with schematic layouts of the facility is given elsewhere. ${ }^{14}$

\section{APPLICATION OF AN EMCCD FOR NUSTAR GROUND CALIBRATION}

All three NuSTAR telescopes (two flight optics and one flight spare) were calibrated at RaMCaF between October 2010 to March 2011. Before characterizing flight telescopes, we verified the linear response of the EMCCD camera by increasing the source current of the Comet XRS-100 x-ray system installed at the calibration facility. We cross-calibrated with a high-purity Germanium detector which was also used for optics characterization since flight detectors were not available at that time.

During measurements of the optics response of all three $N U S T A R$ telescopes the camera was operated in integration mode. For the flight optics calibration, the $3: 1$ taper coupled to a CsI(Tl) scintillator $(450 \mu \mathrm{m}$ thick) was used to cover a larger fraction of the optics response. In order to study the full extent of the image for all NuSTAR optics we mounted the detector on a motorized stage and scanned the telescope output in the plane orthogonal to the beam axis. The scintillator-coupled EMCCD camera played a crucial role in the ground calibration effort. The following procedures were performed using this detector:

- Installation and alignment of $N U S T A R$ telescopes in the beamline of the RaMCaF facility. 

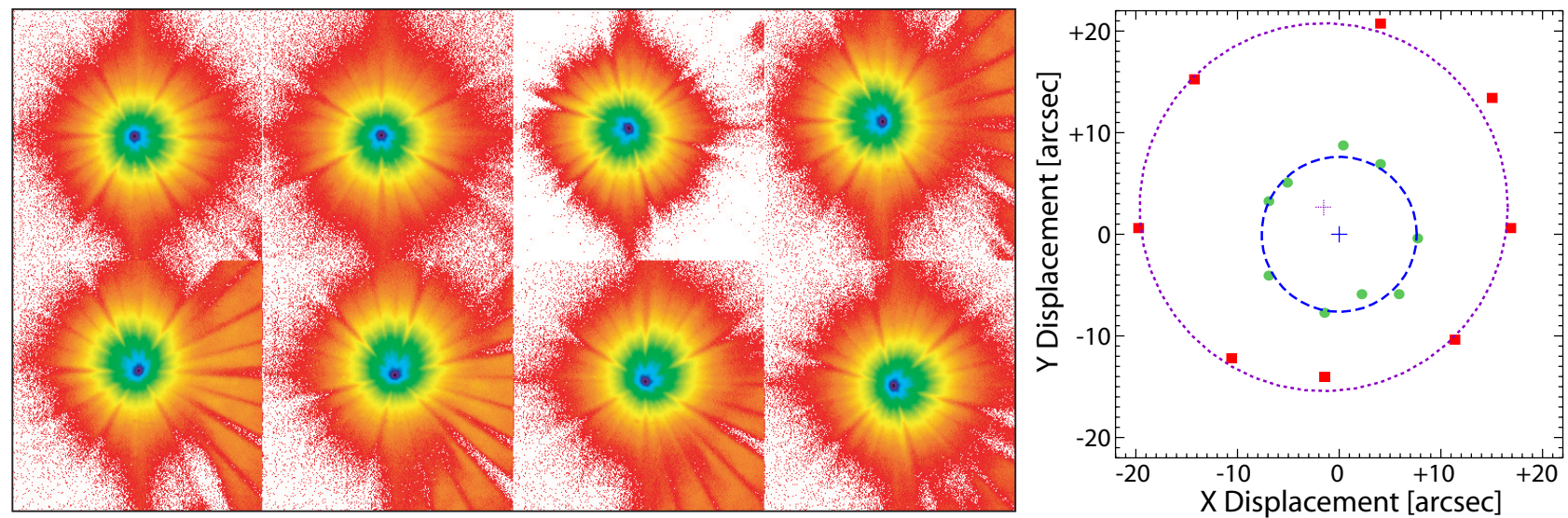

Figure 3. Left: Point spread function (PSF) data acquired when rotating the telescope in increments of $45^{\circ}$ about the axis. The shift of the centroid position in these images indicates that the telescope axis is misaligned with respect to the facility axis. Right: Relative centroid positions of the PSF as a function of various azimuth positions. Red squares and purple dotted circle indicate the initial position, the green circles and blue dashed circle demonstrate the improved alignment after adjustments.

- Characterization of point-spread function (PSF) on-axis and off-axis, in discrete energy bands and the full range spectrum of the calibration facility.

- Validation of ray-trace simulations with respect to effects of finite source distance and the use of a diverging source, as well as the influence of gravity, figure, and finish.

- Characterization of RaMCaF features, such as slit size and position, monitoring through-holes in the experimental setup, etc.

The efficiency of using a silicon solid-state detector coupled to a scintillating material for development and calibration of x-ray optics has been validated with the data acquired as part of the NuSTAR ground calibration campaign. In the following subsections we will discuss each task in more detail.

\subsection{Optics Installation and Alignment}

During calibration, the NuSTAR optics were mounted on a large rotation stage allowing for translations perpendicular to the x-ray axis of the facility as well as movement in roll, pitch and yaw*. A series of adjustable struts kept the optic in place and prevented unwanted movement. Initial alignment of the x-ray axis of the telescope to the x-ray axis of the calibration facility was done using visible light surveying techniques and fiducial alignment features. The proxy for the x-ray axis of $R a M C a F$ were two sets of cross-hairs that consist of two perpendicular wires. The proxy for the x-ray axis of the telescopes were two pinholes located at the front and back of the cylindrical support on which the x-ray telescope was built. The position of the optic was changed (by adjusting both the struts and the location of the rotation stage) until the pinholes were co-located to the cross-hairs.

After initial alignment, the optic was illuminated with x-rays and the centroid of the pinhole was recorded on the EMCCD as a function of azimuthal (or roll angle) position within the large rotation stage. Alignment needed to be validated in situ and improved if necessary. A rapid and effective collection of x-ray data was therefore an important factor. To illustrate the use of the EMCCD detector, Fig. 3 (left) shows images of the PSF at 8 different roll angles. The centroid position for each PSF image was used to quantify the amount of precession (see Figure 3, right). The circles represent the magnitude of the precession between the rotation axis of the large stage to which the $\mathrm{x}$-ray telescope is mounted and the facility x-ray axis. The crosses indicate the center points of the precession circles and any difference from $(0,0)$ indicates an additional offset of the two x-ray axes.

${ }^{*}$ See Ref. ${ }^{14}$ for a detailed description of coordinate systems and motion requirements for the RaMCaF facility. 


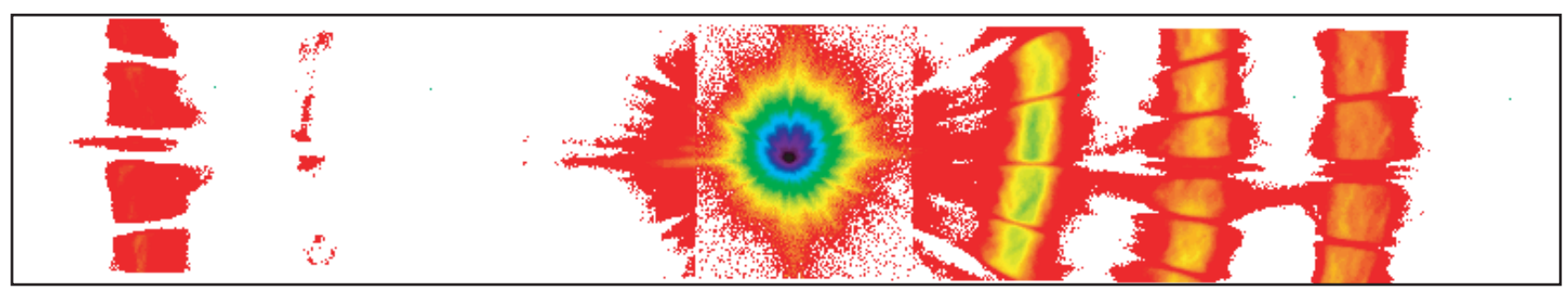

Figure 4. Telescope response for source $2^{\prime}$ off-axis. Image is a composite of data acquired at seven different horizontal positions. The central core shows the properly focused light and is the true PSF. The vertical streaks are an artifact and result from events registered during the readout of the CCD chip. The vertical bands to the left and right of the core (three to the right, two to the left) result from single reflected light and are referred to as ghost-rays. The apparent vertical bands are in reality annular rings, that extended above and below the area of the detector. These rings result from the use of aperture limits used to illuminate specific regions of the telescope.

Linear dimensions were converted to angular displacement using a plate scale of $1 \mathrm{~mm}=19.1^{\prime \prime}$. In this specific example the recorded data showed that the initial alignment (red squares/purple dotted line, $0.94 \mathrm{~mm}\left[18^{\prime \prime}\right]$ radial precession) did not meet the requirement $\left(0.52 \mathrm{~mm}\left[10^{\prime \prime}\right]\right)$. After additional adjustments were made, the PSF measurements at different roll positions were repeated (green circles/dashed line, $0.42 \mathrm{~mm}\left[8^{\prime \prime}\right]$ radial precession) and used to verify that the precession tolerance met specifications. The two data sets show that after the re-adjustment, the alignment was improved and fully met the $N u S T A R$ calibration requirements ${ }^{\dagger}$.

\subsection{Point Spread Function}

Measuring the point spread function (PSF) of the NuSTAR telescopes was one of the main goals of the optics calibration campaign and the major task of the EMCCD detector. This camera made it possible to study the evolution of the PSF as a function of both off-axis position and energy in real time. In order to observe energy dependencies, we created broad spectral bands by precisely matching the end-point energy of the x-ray source with carefully chosen filters. A total of four energy bands were studied: a broad band ${ }^{\ddagger}$ and three narrow Gaussian-like bands of about $15 \mathrm{keV}$ width centered on 30,55 and $70 \mathrm{keV}$. In order to additionally determine the off-axis response of the telescopes, the pitch and yaw of the optics' mounting were carefully adjusted.

It is important to mention that the measured performance on ground will differ from the expected performance in space. This is due to several factors: the distance between the x-ray source and the telescope is finite, the beam is divergent and the stray-light-limiting apertures that will be present in the satellite are absent on ground. Therefore additional features of the PSF, which will not be observable when NuSTAR is in orbit, are expected to appear in groundbased calibration measurements The most important effect observable on ground are "ghost rays" resulting from photons that have only been reflected off a single mirror surface. The core of the PSF on the other hand is formed be double reflected photons that are properly focused. While ghost rays are unwanted features on orbit, they can be utilized to identify figure errors in individual mirror segments during ground calibrations.

An example showing ghost rays can be found in Figure 4. The image shows a sub-group illumination measurement during which only limited regions of the optics (subgroups) were illuminated with x-rays. It was obtained by systematically moving the camera in the horizontal direction in seven different position, because the ghost rays extend well beyond the field of view of the EMCCD chip (about $25 \mathrm{~mm} \times 25 \mathrm{~mm}$ ). The resulting mosaic image was corrected for different exposure times in different areas of the image. The total image is approximately $150 \mathrm{~mm}$ wide.

In order to obtain the PSF of the entire telescope, the optics were systematically rotated about their optical axis and horizontal raster-scans were acquired at each rotation position. The images of these different positions were then assembled and corrected for different exposure times. For subgroup illumination of the full optic (NuSTAR flight module 1, FM1), 70 images of EMCCD data were acquired. For full-flood illumination (NuSTAR

\footnotetext{
${ }^{\dagger}$ Note that the observable asymmetry in the images results from a combination of two effects: the influence of gravity and a bend of the optics due to stress induced by tightened mounting struts of the telescope support.

${ }^{\ddagger}$ The full broad band consists of a bremsstrahlung spectrum of the source spanning energies from about 15-100 keV with target material lines at the higher end of the spectrum.
} 
flight module 2, FM2), 90 exposures were used to create a view of the full optics. The resulting mosaic images are shown in the left and right part of Figure 5, respectively. Note that the color scale is logarithmic in these plots, i.e. ghost rays are very faint compared to the core image. The shadow effect of the telescope spider can be identified. Individual graphite spacers separating the single pieces of glass can be seen. The left part of Figure 5 depicts the illumination of 4 subgroups. In the full flood illumination (Fig. 5 , right), the change from $60^{\circ}$ azimuthal sectors (inner mirror shells) to $30^{\circ}$ sectors (outer shells) is observable.

\subsection{Validation of Raytrace Simulations}

In order to predict on-orbit telescope performance, an accurate optics model is required. Such a model must be able to predict differences resulting from distinct sources and it has to reproduce results from ground calibrations. Astrophysical and facility sources differ significantly: while astrophysical sources provide parallel light filling the optics' aperture uniformly, the light originating from calibration sources is both divergent and spatially extended such that there might not be a homogeneous illumination of the mirror surfaces or the telescope aperture.

The common approach for x-ray telescopes, is to first use a Monte Carlo simulation to generate individual photons and then propagate each generated photon through the telescope. This ray-tracing technique, accounts for various factors including finite reflectivity, figure errors, spatial roughness and the influence of support structures. Performance factors (e.g. mirror roughness or obscuration percentages) can either be adjusted to match the calibration data or they can be fixed, based on previous measurements. The NuSTAR telescope model and the complete simulators NuSim and MT-Rayor are discussed in detail in Ref. ${ }^{15,16}$

At $N u S T A R$ data aquired with the EMCCD was used to validate the NuSIM raytrace model. Figure 6 provides an example, in which an off-axis data sample is compared to a simulation run. The main features of the data set are well reproduced by the model. A comparison of these data with the more accurate simulator MT-Rayor is work in progress.
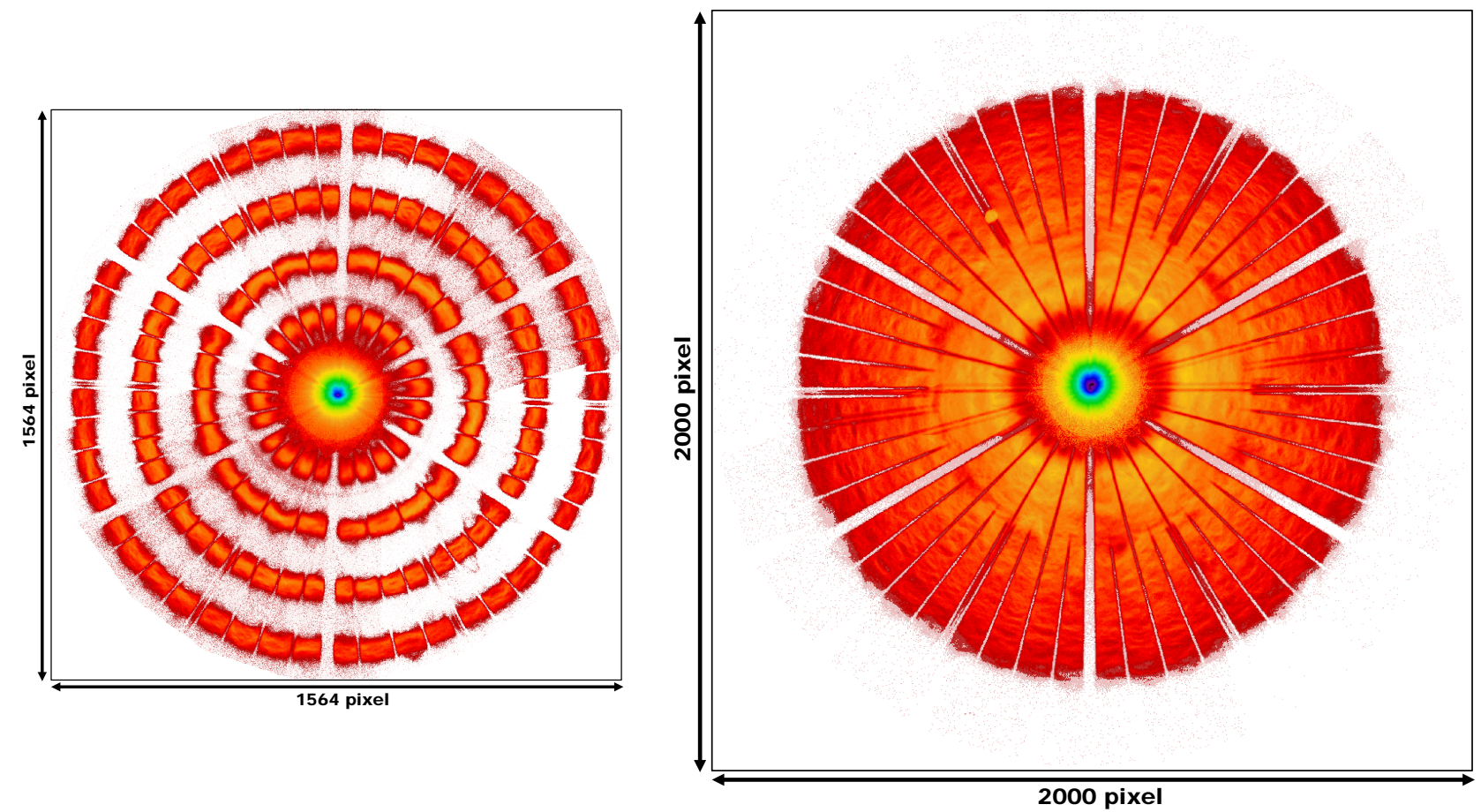

Figure 5. Left: Illumination of subgroups for NuSTAR's FM1 optic, obtained by mosaic imaging with the EMCCD camera. Four subgroups were illuminated here. The spider structure of the optics support is visible in the logarithmic color scale display. Right: Full flood illumination of NuSTAR's FM2 optic, obtained by mosaic imaging with the EMCCD camera. Both the spider structure and the graphite spacers can be observed in this image. Note the logarithmic color scale used. 

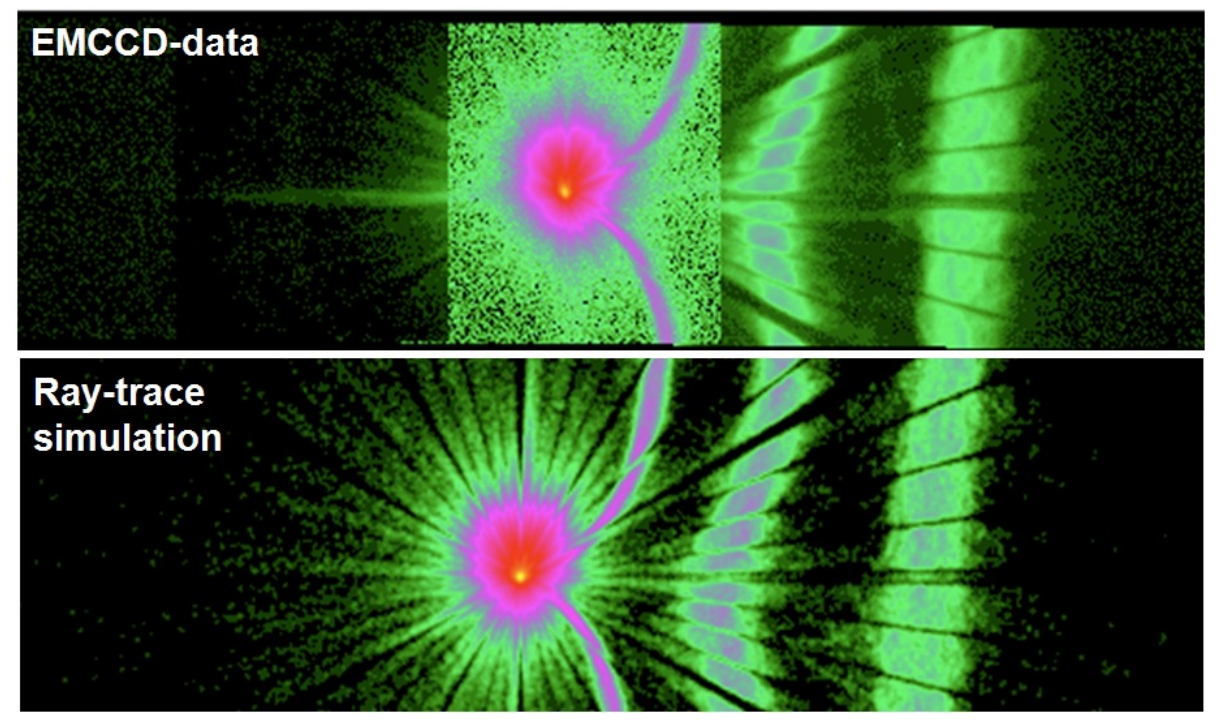

Figure 6. Comparison between data acquired at RaMCaF with one of the NuSTAR optics using the EMCCD and a $N u S I M$ raytrace simulation. Data were acquired in an off-axis position to validate this raytrace model.

\subsection{Characterization of Facility Features}

During NUSTAR ground calibration, the EMCCD detector was also used for another important task: a characterization of the x-ray calibration facility. The nominal behavior of x-ray source and other equipment can be verified before, during and after the actual measurements by using the EMCCD detector. This helps to justify the assumption that during optics calibration all components behave as intended. Since the camera was mounted on translation stages such that it could be moved over large ranges, it was able to map the illumination pattern of the x-rays originating from the source ensuring they have the desired properties. As an example, Figure 7 shows a data set that was used to identify that a drive mechanism had not been retracted properly. It partially obscured the entrance aperture and in this case imaging with a 2D detector allowed for fast and unambiguous diagnosis of the situation.

\subsection{Limitations of the scintillator-coupled EMCCD}

The results of the NuSTAR calibration campaign with the EMCCD detector system showed the advantages of using such an easy-to-operate $2 \mathrm{D}$ detector with high spatial resolution and low noise. However some limitations in the energy range of $5-25 \mathrm{keV}$ became apparent and the detector performance in this soft x-ray range was suboptimal. This was in part due to the use of a rather thick scintillator $(450 \mu \mathrm{m})$ for the conversion of x-rays into visible photons. The thickness was chosen to optimize the detector performance at higher energies $(25-80 \mathrm{keV})$. Most low energy x-rays were absorbed close to the entrance surface, i.e. within the first $100 \mu \mathrm{m}$. Self-absorption of the scintillation light in the $\mathrm{CsI}(\mathrm{Tl})$ scintillator occured. Using a thin scintillator of $150 \mu \mathrm{m}$ would improve the performance at low energies, but requires a switch of scintillator when changing to higher energies. In order to improve the performance of the detector over a larger range of energies, the characteristics of the scintillator have to be improved.

\section{METHODS TO IMPROVE THE EMCCD DETECTOR PERFORMANCE}

To optimize the detector system for optics calibration of future missions several methods to improve its performance are being investigated. The upgrades include both hardware and software improvements and are outlined in the following. 

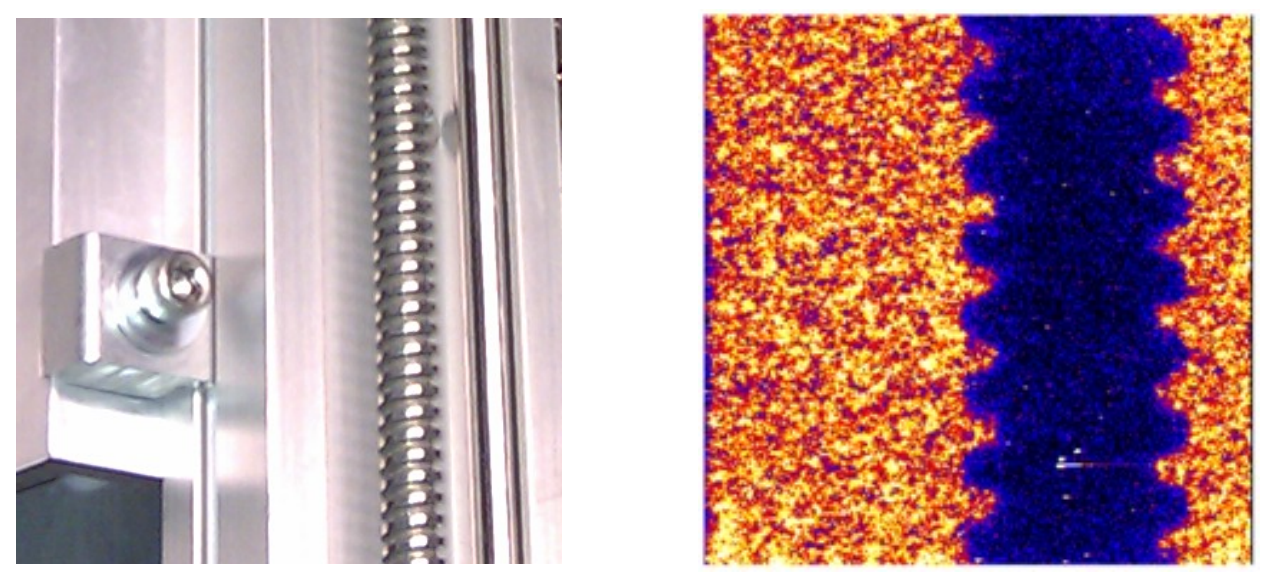

Figure 7. Left: Photo of the drive screw (right part of image). Right: Shadow originating from a drive screw that was not retracted properly and was thus blocking the line of sight for the detector.

\subsection{Hardware Improvements}

Three major upgrades to the hardware system are envisioned for our future work. One approach will focus on improving the performance of the detector by upgrading the EMCCD camera, while the second aim is to enhance the fiber optic bonding to the scintillator. The third envisioned change to the system will be refining the choice of scintillator material itself.

\section{EMCCD Camera}

The next generation detector under development uses an Andor iXon DU888 EMCCD. This next-generation EMCCD has a larger active area, a smaller pixel size and a higher quantum efficiency than the detector used during NuSTAR calibrations yielding an improvement in signal to noise ratio (SNR) for single photon x-ray detection. A resolution of one megapixel with a pixel size of $13 \times 13 \mu \mathrm{m}$ is expected and yields a total detector area of $13.3 \times 13.3 \mathrm{~mm}^{2}$ without fiberoptic taper. The estimated SNR to detect single $8 \mathrm{keV}$ photons with this detector system using a $150 \mu \mathrm{m}$ thick $\mathrm{CsI}(\mathrm{Tl})$ scintillator is $\mathrm{S} / \mathrm{N}=19$ for a $1: 1$ taper and $\mathrm{S} / \mathrm{N}=6.7$ for a $2: 1$ taper. ${ }^{17}$

\section{Fiberoptic Coupling}

The detector used for calibration measurements of $N u S T A R$ consisted of a $450 \mu \mathrm{m}$ thick $\mathrm{CsI}(\mathrm{Tl})$ scintillator that was coupled via a fixed 1:1 optical plug and an additional 3:1 fiberoptic taper to the back-thinned EMCCD chip. For the next generation of the device, the 1:1 plug will be omitted and the small end of the 3:1 taper will be directly bonded to the EMCCD chip. Since each interface results in losses of spatial resolution and signal, this is a promising way to minimize signal degradation and simultaneously enhance sensitivity and energy resolution. The deposition process to obtain a uniform columnar structure of the scintillator directly on the fiberoptic taper differs from the standard process used for deposition on thin graphite substrates due to the larger mass and size of the taper. Alteration of the thermodynamics of the deposition process due to the low thermal conductivity of the fiberoptic glass leads to a lower light yield for the taper than for the graphite substrate since an amorphous layer is formed on the taper surface. This issue can be addressed by optimizing the deposition conditions.

\section{Scintillator}

While the straightforward solution to improve the detector performance at different energies is to use scintillators of varying thickness that are matched to the energy that is being measured, i.e. thin materials for low energy $\mathrm{x}$-rays and thicker ones for higher energies, an optimization of the scintillator itself is necessary in order to achieve improved performance over the entire energy range of interest.

Studies were performed to change the structure of the microcolumnar $\mathrm{CsI}(\mathrm{Tl})$ from the commonly used 
amorphous microcolumnar structure (AMS) to a crystalline microcolumnar structure (CMS). This can be accomplished by a change of the deposition parameters and resulted in brighter films with slightly more light spread. Due to the higher brightness the CMS CsI(Tl) remains a possible alternative for the standard AMS films.

Improvement can also originate from using a different scintillator material with higher light yield. Thalliumdoped $\mathrm{CsI}[\mathrm{CsI}(\mathrm{Tl})]$ has traditionally been the favored material due to its suitable scintillation properties in combination with the advanced deposition technology available to manufacture the microcolumnar structure of the scintillator film. Materials that are being considered for future application include $\mathrm{Ba}_{2} \mathrm{CsI}_{5}(\mathrm{Eu})$. This relatively new scintillator has very favorable scintillation properties such as its brightness. While $\mathrm{CsI}(\mathrm{Tl})$ emits about 60,000 photons $/ \mathrm{MeV}, \mathrm{Ba}_{2} \mathrm{CsI}_{5}(\mathrm{Eu})$ can increase this number by a factor of more than 1.6. Studies of appropriate deposition processes for the new scintillator are already under way.

\subsection{Software Upgrades}

Based on measurements using two radioactive sources (Am-241 and Cd-109), a prototype algorithm to detect single photon events has been developed. Preliminary results indicate that higher energy photons can be detected when using a rather thick scintillator $(450 \mu \mathrm{m})$, while for photons below about $25 \mathrm{keV}$ similar results are not achievable in this configuration but should yield comparable results if a thinner scintillator is used. The lower energy threshold remains yet to be determined.

Future work will focus on several tasks. This will include improving the single-photon counting algorithms by making it more robust. Some improvement in software speed will also be addressed, since this will facilitate the handling of larger volumes of data. Generally several hundred frames are taken per measurement. This number of frames would likely have to be increased (in comparison with operation in integration mode) due to the fact that the count rate per frame is limited since overlapping hits are extremely difficult to detect as separate events. An upper limit on the maximal count rate per data frame will be determined along with the low energy threshold achievable for various scintillators. Further work will also improve the determination of deposited energy and thus enhance the energy resolution.

\section{CONCLUSIONS AND OUTLOOK}

In this paper, we demonstrated the potential of a scintillator-coupled EMCCD camera for calibrating hard x-ray telescopes for future satellite missions by presenting the results achieved during the ground calibration of the $N u S T A R$ flight optics. The detector was used to align the telescopes and to measure the point spread function as well as for validating the optics model.

The goal for the improved detector system currently under construction is to provide a high spatial resolution of about $25 \mu \mathrm{m}$ over the full energy range of interest (about $5-100 \mathrm{keV}$ ), while having at the same time a good energy resolution, a large active area $\left(\sim 25 \mathrm{~cm}^{2}\right)$, high rate capability and a flexible design that is easy to operate. Various methods to upgrade the scintillator-coupled EMCCD are under investigation and include hardware improvements of all major detector components including the EMCCD detector, the fiberoptic coupling and the scintillator material. On the software side, the development of single-photon counting algorithms is expected to improve the performance by enhancing the energy resolution. We expect these upgrades to render the detector even more suitable for hard x-ray optics calibrations than the prototype and simplify energy-dependent studies of effective area and PSF.

\section{Acknowledgment}

This work was performed under the auspices of the U.S. Department of Energy by Lawrence Livermore National Laboratory under Contract DE-AC52-07NA27344. The support of the Laboratory Directed Research and Development Program is gratefully acknowledged. We thank our colleagues at NuSTAR for their support and cooperation and Columbia University for hospitality. We also thank NASA for funding this research under grant number 


\section{REFERENCES}

[1] Harrison, F. A. et al., "Development of the HEFT and NuSTAR focusing telescope," Exp. Astron 20, 131-137 (2005).

[2] Ramsey, B. D. et al., "First Images from HERO, a Hard X-Ray Focusing Telescope," APJ 568, 432-435 (2002).

[3] Tueller, J. et al., "InFOC $\mu$ S Hard X-Ray Imaging Telescope," Exp. Astron. 20, 121-129 (2005).

[4] Harrison, F. A. et al., "The Nuclear Spectroscopic Telescope Array (NuSTAR)," Proc. SPIE 7732, 27 (2010).

[5] Takahashi, T. et al., "The ASTRO-H Mission," Proc. SPIE 7732,- (2010).

[6] http://www.andor.com/scientific_cameras/ixon_emccd_camera/.

[7] Nagarkar, V. V. et al., "Fast X-Ray/ -Ray Imaging using Electron Multiplying CCD-Based Detector," NIM A 563, 45-48 (2006).

[8] Nagarkar, V. V. et al., "Structured Csi(Tl) Scintillators for X-Ray Imaging Applications," IEEE Trans Nucl. Sci 45, 492-496 (1998).

[9] Hailey, C. J. et al., "The Nuclear Spectroscopic Telescope Array (NuSTAR): Optics Overview and Current Status," Proc. SPIE 7732, $77320 \mathrm{~T}$ (2010).

[10] Craig, W. et al., "Fabrication of the NuSTAR Flight Optics," Proc. SPIE 8147, 81470H (2011).

[11] Christensen, F. et al., "Coatings for the NuSTAR Mission," Proc. SPIE 8147, 81470 U (2011).

[12] Pivovaroff, M. J. et al., "The nuclear spectroscopic telescope array, NuSTAR: mission overview," Proc. SPIE 8443, 69 (2012).

[13] Brejnholt, N. F. et al., "NuSTAR on-ground calibration I: effective area," Proc. SPIE 8443, 70 (2012).

[14] Brejnholt, N. F. et al., "The Rainwater Memorial Calibration Facility for X-Ray Optics," X-Ray Optics and Instrumentation 2011, 285079 (2011).

[15] Zoglauer, A. et al., "Simulating Extended Galactic Sources with the NuSTAR Simulator NuSIM," BAAS 43, 6, 43.07 (2011).

[16] Westergaard, N. J. S. et al., "NuSTAR on-ground calibration II: imaging quality," Proc. SPIE 8443, 68 (2012).

[17] Kudrolli, H. et al., "Development of high spatial resolution detector for characterization of X-ray optics," JINST 6, C12013 (2011). 